\title{
Massive MIMO as a Cyber-Weapon
}

\author{
Marcus Karlsson and Erik G. Larsson
}

\section{Linköping University Post Print}

\section{Tweet}

N.B.: When citing this work, cite the original article.

Original Publication:

Marcus Karlsson and Erik G. Larsson, Massive MIMO as a Cyber-Weapon, 2014, 48th Asilomar Conference on Signals, Systems and Computers, 661-665.

http://dx.doi.org/10.1109/ACSSC.2014.7094529

Copyright: (C2014 IEEE. Personal use of this material is permitted. However, permission to reprint/republish this material for advertising or promotional purposes or for creating new collective works for resale or redistribution to servers or lists, or to reuse any copyrighted component of this work in other works must be obtained from the IEEE.

\section{http://ieeexplore.ieee.org/}

Postprint available at: Linköping University Electronic Press

http://urn.kb.se/resolve?urn=urn:nbn:se:liu:diva-128271 


\title{
Massive MIMO as a Cyber-Weapon
}

\author{
Marcus Karlsson and Erik G. Larsson
}

\begin{abstract}
A scheme for a multi-antenna jammer, jamming a SISO primary link working in TDD mode is presented. The jammer relies on reciprocity to estimate the channel to a terminal in the primary link blindly. The jammer can then beamform noise towards this user to degrade the performance of the primary link. Simulations show that, for certain scenarios, the proposed jammer can do the same damage to the primary link as an omnidirectional jammer, but with close to two orders of magnitude lower output power.
\end{abstract}

\section{BACKGROUND}

Massive MIMO refers to using large numbers, a hundred or more, of phase-coherently operating antennas at a wireless base station. This technology, originally conceived by Marzetta [1] is a game-changer in wireless access technology and has created a research area in which large progress has been made recently and which has attracted substantial academic and industrial sponsorship [2], [3]. Two tutorials that outline the main features of massive MIMO technology are [4], [5]. A paper that quantifies the potential of massive MIMO in a realistic multi-cell setup is [6].

The basic presumption of massive MIMO is that the base station can estimate the channel response to the terminals being served, by using measurements on uplink pilots. By exploiting channel reciprocity, these channel responses are then used in the downlink to perform multiuser beamforming. One of the main insights from the massive MIMO literature is that with $M$ antennas, and estimated channels, the transmitted power (both on uplink and downlink) can be scaled down as $O(1 / \sqrt{M})$ at a fixed information rate. Hence, when increasing $M$, the quality of the estimated channel state information gets worse and worse (because the SNR scales proportionally to $1 / \sqrt{M}$ ), but this does not matter: the excess of transmitter antennas more than compensates for this loss of SNR. There is also substantial evidence that massive MIMO can be built with low-cost (low-accuracy) hardware [7]; hardware-induced distortion adds up "non-coherently" and the excess of transmitter antennas compensates for the increased hardware noise. Complexity-wise, massive MIMO with conjugate beamforming and TDD operation is entirely scalable: complexity (the computational operations and the amount of digital and RF hardware needed) is proportional to $M$.

Prototype implementations of massive MIMO already exist. The Argos testbed [8] has been successfully used to demonstrate that the beamforming gains predicted by theory hold true in practice, and additionally, that after appropriate

M. Karlsson and E. G. Larsson are with the Department of Electrical Engineering (ISY), Linköping University, 58183 Linköping, Sweden (email: \{marcus.karlsson, erik.g.larsson\}@liu.se).

This work was supported in part by the Swedish Research Council (VR), and ELLIIT. calibration, the assumptions on channel reciprocity hold. In the experiments documented in [8], 96 antennas were used. Another testbed underway [2] aims at using 100 antennas at a single base-station. Given current investments in massive MIMO (e.g., [2], [3]) it is likely that in a not-too-distant future, we will see many testbeds of this sort in full-scale operationperhaps with even more antennas.

\section{A. Vulnerable Wireless: the Jamming Threat}

Jamming of wireless links represents a rapidly increasing, but seemingly less known cyber-security threat. Some examples are: jamming of private mobile radio (PMR) systems, used by police and other first responder personnel; jamming (and spoofing) of GPS receivers [9]; jamming of car lock remote controls in parking lots to facilitate easy theft of property; and jamming of wireless alarm systems in single-family homes to enable undetected burglary. Simple wireless jammers can be bought off the Internet for a few $\$ 100$, and equipment that used to be military-grade can be put together using off-the-shelf software radio-based platforms for a few $\$ 1000$. Numerous recent incidents, especially in public safety applications, illustrate the magnitude of the problem. During the EU summit in Gothenburg, Sweden, in 2001, activists used (illegal) jamming equipment located in a nearby apartment and during critical phases of the riots, the chief commander could not reach any of the 700 engaged police officers [10]. In addition, jamming against LTE, when used for PMR applications. is a growing concern [11].

Traditionally, jamming of wireless links has been considered a "military" problem, even though the awareness of the threat to civilian systems seems to be increasing. There may be a parallel with cyber-security on the Internet. Consider D-DoS attacks. They were not foreseen in the 1990's-at least, the Internet and its protocols and servers were clearly not designed with this threat in mind - but this treat is now widespread and taken very seriously at all levels. It is not implausible that wireless jamming can undergo the same evolution, because: the harm that can be caused is substantial; society is relying more and more on wireless communications solutions; inexpensive, military-grade technology is proliferating; and the ability of an adversary to hide or to operate covertly is increased, for example due to the availability of cheap unmanned aerial vehicles (UAVs).

\section{B. Contribution of the Paper}

We demonstrate that if massive MIMO technology falls in the hands of adversaries, it can be used as a jamming device and cause significant harm to conventional wireless 
communication links that operate in TDD mode. The adversary must know the slot duration, but needs no other knowledge of the system to be jammed (such as channel state information, timing synchronization, pilot sequences or codebooks). Also, no "rank assumptions" on the propagation channels are needed. A TDD system is fundamentally sensitive to jamming attacks, because during transmission mode, it reveals its "location" so that an adversary could estimate the transmit strategy that would cause maximal harm. Some PMR systems-for example TETRA, used for police and firefighter communications-operate in TDD mode and hence are exposed to the vulnerability that we demonstrate here.

We quantity the extent of the harm that can be caused as functions of some key system parameters: number of antennas at the jammer; coherence time (determined by mobility); slot timing (determined by design of the primary system, must be less than the coherence time); and the distance between the primary system and the jammer.

\section{SCENARIO}

Conventional jammers spread the jamming signal omnidirectionally in order to jam a particular target. This is highly inefficient, because most of the output power will not reach the intended target and is therefore wasted. Since only a small portion of the output power is destructive to the target, very large output powers are generally needed. This huge output power and omnidirectional output makes the this type of jammer relatively easy to locate.

Directional jammers, such as the one presented in this paper, will need much less output power to be destructive to the primary link, due to the large number of antennas, $M$. The $M$ antennas will make the jammer able to beamform "noise" to the target which provides an array gain over the omnidirectional jammer. Owing to the decrease in output power and the fact that most of the signal power is directed towards the target, a directional jammer is much more difficult to locate.

\section{A. Primary System Transmission Scheme}

Consider a system with two single antenna terminals, A and $\mathrm{B}$, communicating in TDD mode over a narrowband, quasi-static channel with gain $h \sim \mathcal{C N}(0,1)$. The coherence time $T_{C}$, over which the channel is essentially constant, is split equally between the two terminals. With a coherence bandwidth of $B_{C}$, the coherence interval is $\tau_{C}=T_{C} B_{C}$ complex samples.

The transmission scheme for the primary link can be seen in Figure 1. The $k$ :th coherence interval is divided into two frames, $A_{k}$ and $B_{k}$. During frame $A_{k}$ terminal A transmits $\tau_{C} / 2$ symbols, and during frame $B_{k}$ terminal B transmits $\tau_{C} / 2$ symbols.

$$
\begin{array}{|l|l|l|l|l|l|l|l|l|l|}
\hline A_{1} & B_{1} & A_{2} & B_{2} & \cdots & A_{k} & B_{k} & \cdots & A_{K} & B_{K} \\
\hline
\end{array}
$$

Fig. 1. The transmission structure for the TDD link between A and B. Each coherence block $k$ is divided into two frames, $A_{k}$, where $\mathrm{A}$ is transmitting, and $B_{k}$, where $\mathrm{B}$ is transmitting.
Let $T_{0} \in \mathbb{N}$ denote the frame timing, the time (sample) when communication between $\mathrm{A}$ and $\mathrm{B}$ begins. The $k$ :th coherence block is divided into $A_{k}$ and $B_{k}$ as

$$
\begin{aligned}
& t \in A_{k} \Leftrightarrow t-T_{0} \in\left[(k-1) \tau_{C},(k-1) \tau_{C}+\tau_{C} / 2-1\right], \\
& t \in B_{k} \Leftrightarrow t-T_{0} \in\left[(k-1) \tau_{C}+\tau_{C} / 2,(k-1) \tau_{C}+\tau_{C}-1\right] .
\end{aligned}
$$

The purpose of the jammer is to disrupt communication in one direction, say $\mathrm{B} \rightarrow \mathrm{A}$. This is done by beamforming noise to terminal A while terminal $\mathrm{B}$ is transmitting. In order to be able to beamform, the jammer must first estimate the channel to A, $\boldsymbol{g}_{A}$. This has to be done every coherence interval. First, however, the jammer must estimate the frame timing, $T_{0}$.

\section{PROPOSED JAMMER}

The general structure of the jamming scheme was mentioned in Section II-A and is explained in more detail here. To be able to jam the primary link, the jammer must:

1) Estimate the frame timing, $T_{0}$

2) Estimate the channel to terminal $\mathrm{A}, \boldsymbol{g}_{A}$

3) Beamform noise to terminal $\mathrm{A}$

To estimate the channel to terminal $\mathrm{A}$, the jammer relies on reciprocity; the channel from $\mathrm{A}$ to the jammer and the channel from the jammer to $\mathrm{A}$, will be the same within a coherence block. This means that once B starts transmitting, the jammer can beamform noise in the direction of the channel it learned in the previous frame. The jammer has to estimate the channel every coherence block, while the frame timing is only estimated once.

\section{A. Estimating the Frame Timing}

Accurately estimating the frame timing is key to be able do disrupt the primary link as much as possible. A poorly estimated $T_{0}$ will have two major effects, both of which degrades the jammer's performance. The first is a "degree of freedom" loss, as the jammer loses jamming opportunity; the second is a degradation in the accuracy of the channel estimate. This will be explained in more detail in the end of Section III-A.

Since the transmitter in the primary link changes every frame, the channel the jammer sees will effectively change every frame. This change is what the jammer utilizes in order to estimate the frame timing. The $M \times 1$ received signal at the jammer in frame $F$ is:

$$
\boldsymbol{y}(t)=\boldsymbol{g} x(t)+\boldsymbol{w}(t)
$$

where $x(t) \sim \mathcal{C N}\left(0, \rho_{p}\right)$ is the transmitted primary symbol at time $t, \boldsymbol{g} \sim \mathcal{C N}(0, \boldsymbol{I})$ is the channel, and $\boldsymbol{w} \sim \mathcal{C N}(0, \boldsymbol{I})$ is white (thermal) noise. To make notations easier, it is assumed that both of the primary terminals transmit with the same output power, $\rho_{p}$. To estimate $T_{0}$, the jammer listens to $K$ coherence blocks. Define the set of all frames $\mathcal{F}=\cup_{k=1}^{K}\left(A_{k} \cup B_{k}\right)$ and let $\boldsymbol{g}_{F}$ be the channel realization in frame $F$. Furthermore, let $\boldsymbol{y}_{F}(t)=\boldsymbol{y}(t), t \in F$, i.e. the received signal in frame $F$. 
Now, given the channel in frame $F \in \mathcal{F}$, the probability density function of the received signal in frame $F$ is

$$
p_{\boldsymbol{y}_{F}}\left(\boldsymbol{y} \mid T_{0}, \boldsymbol{g}_{F}\right)=\frac{1}{(2 \pi)^{M}\left|\boldsymbol{Q}_{F}\right|} \exp \left(-\boldsymbol{y}^{\mathrm{H}} \boldsymbol{Q}_{F}^{-1} \boldsymbol{y}\right)
$$

where $\boldsymbol{Q}_{F}=\mathbb{E}\left[\boldsymbol{y}_{F} \boldsymbol{y}_{F}^{\mathrm{H}}\right]=\rho_{p} \boldsymbol{g}_{F} \boldsymbol{g}_{F}^{\mathrm{H}}+\boldsymbol{I}$. The likelihood function of $T_{0}$ for frame $F$ is

$$
L_{F}\left(T_{0} \mid \boldsymbol{y}_{F}(t), \boldsymbol{g}_{F}\right)=\prod_{t \in F} p_{\boldsymbol{y}_{F}}\left(\boldsymbol{y}(t) \mid T_{0}, \boldsymbol{g}_{F}\right)
$$

To estimate $T_{0}$ the jammer listens for $K$ blocks and constructs the likelihood-function

$$
L\left(T_{0} \mid \boldsymbol{y}(t),\left\{\boldsymbol{g}_{F}\right\}_{F \in \mathcal{F}}\right)=\prod_{F \in \mathcal{F}} L_{F}\left(T_{0} \mid \boldsymbol{y}_{F}(t), \boldsymbol{g}_{F}\right) .
$$

Maximizing (5) is equivalent to minimizing

$$
\begin{aligned}
& l\left(T_{0} \mid \boldsymbol{y}(t),\left\{\boldsymbol{g}_{F}\right\}_{F \in \mathcal{F}}\right) \triangleq-\log L\left(T_{0} \mid \boldsymbol{y}(t),\left\{\boldsymbol{g}_{F}\right\}_{F \in \mathcal{F}}\right) \\
& =\sum_{k=1}^{K}\left(\sum_{t \in A_{k}}\left(\log \left|\boldsymbol{Q}_{A_{k}}\right|+\boldsymbol{y}(t)^{\mathrm{H}} \boldsymbol{Q}_{A_{k}}^{-1} \boldsymbol{y}(t)\right)\right. \\
& \left.+\sum_{t \in B_{k}}\left(\log \left|\boldsymbol{Q}_{B_{k}}\right|+\boldsymbol{y}(t)^{\mathrm{H}} \boldsymbol{Q}_{B_{k}}^{-1} \boldsymbol{y}(t)\right)\right)+ \text { constant. }
\end{aligned}
$$

The ML estimate of $T_{0}, \hat{T}_{0}$ is then found as

$$
\hat{T}_{0}=\left.\underset{T_{0}}{\operatorname{argmin}} l\left(T_{0} \mid \boldsymbol{y}(t),\left\{\boldsymbol{g}_{F}\right\}_{F \in \mathcal{F}}\right)\right|_{\boldsymbol{g}_{F}:=\hat{\boldsymbol{g}}_{F}},
$$

where $\hat{\boldsymbol{g}}_{F}$ is the jammer's estimate of the channel in frame $F$. How the jammer estimates the channel is described in Section III-B. Since $\boldsymbol{Q}_{F}$ is unknown, the estimate $\hat{\boldsymbol{Q}}_{F}$ is used instead. For a given, hypothetical $T_{0}$, the estimate of $\boldsymbol{Q}_{F}$ is the sample covariance matrix

$$
\hat{\boldsymbol{Q}}_{F}=\frac{1}{|F|} \sum_{t \in F} \boldsymbol{y}(t) \boldsymbol{y}(t)^{\mathrm{H}},
$$

where $|F|$ denotes the number of samples in frame $F$.

Note that the log-likelihood function in (6) will be (almost) periodic, since the jammer can only estimate the frame timing modulo $\tau_{C} / 2$. The period of (6) will be $\tau_{C} / 2$ samples, since that is the length of one frame. What parameters in the simulation that affect the likelihood-function will be covered in Section IV.

Remark: The accuracy of estimating $T_{0}$ will depend on the distance between terminal A and the jammer, as well as the output power of the primary link, $\rho_{p}$, since these factors determine the SNR of the received signal at the jammer. A higher output power will increase the achievable rate of the primary link, but also make the channel easier for the jammer to estimate.

Once $T_{0}$ is estimated, the jammer uses $\hat{T}_{0}$ to calculate the timing of all the frames according to (1), but with $T_{0}$ replaced with its estimate, $\hat{T}_{0}$. The estimate of frame $F$ is denoted $\hat{F}$, see Figure 2.

The quality of the estimate of the frame timing $T_{0}$ determines the overlap between each frame and its corresponding estimate. As an example, assume we only have one coherence block, divided into $A$ and $B$. Furthermore, let $\hat{A}$ and $\hat{B}$ be the corresponding frame estimates and let $\bar{A}=A \cap \hat{A}$ and $\bar{B}=B \cap \hat{B}$. If $T_{0} \neq \hat{T}_{0}$ the estimated frames and the correct frames do not coincide, i.e., $\hat{A} \neq A$ and $\hat{B} \neq B$ as seen in Figure 2.

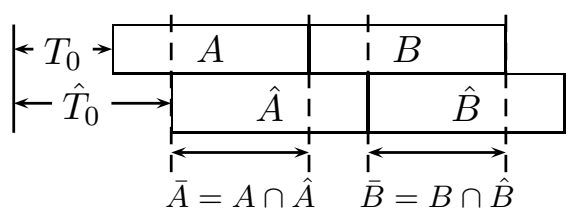

Fig. 2. The difference between the actual frame timings ( $A$ and $B$ ), and the frame timings estimated by the jammer, $\hat{A}$ and $\hat{B}$.

$\bar{A}$ is the part of $\hat{A}$ during which the jammer listens to the correct terminal; the smaller $\bar{A}$ is, the worse the channel estimate will be. Similarly, $\bar{B}$ is the part of $\hat{B}$ during which the jammer beamforms to terminal $A$ when terminal $B$ is transmitting. The smaller $\bar{B}$ is, more time will be spent on trying to jam terminal A, when it is actually transmitting.

\section{B. Estimating the Channel}

The covariance matrix of the received signal in frame $F$ is $\boldsymbol{Q}_{F}=\rho_{p} \boldsymbol{g}_{F} \boldsymbol{g}_{F}^{\mathrm{H}}+\boldsymbol{I}$. This matrix has the eigenvalues $1+\rho_{p} \boldsymbol{g}_{F}^{\mathrm{H}} \boldsymbol{g}_{F}, 1,1, \ldots, 1$, and the eigenvectors corresponding to the eigenvalue $1+\rho_{p} \boldsymbol{g}_{F}^{\mathrm{H}} \boldsymbol{g}_{F}$ is $c \boldsymbol{g}_{F}$, for any $c \in \mathcal{C}$. The channel estimate $\hat{\boldsymbol{g}}_{F}$ is given by the eigenvector corresponding to the dominant eigenvalue of $\hat{\boldsymbol{Q}}_{\hat{F}}$. The scalar ambiguity can be ignored in this case, as the jammer beamforms white Gaussian noise, the phase of the channel vector does not matter. During $\hat{B}_{k}$, the jammer will transmit $x_{J}=\sqrt{\rho_{J}} \frac{\hat{\boldsymbol{g}}_{\hat{A}_{k}}^{*}}{\left\|\hat{\boldsymbol{g}}_{\hat{A}_{k}}\right\|} s_{J}$, where $\rho_{J}$ is the transmit power of the jammer, and $s_{J} \sim \mathcal{C N}(0,1)$ are the noise symbols.

\section{How Good is the Jammer?}

To assess how good the proposed jammer is, a performance metric is needed. The metric chosen is the upper bound on ergodic capacity, which assumes coding across multiple coherence intervals:

$$
\begin{aligned}
R_{\mathrm{B} \rightarrow \mathrm{A}} & =\frac{T_{\bar{B}}}{T_{B}} \mathbb{E}\left[\log _{2}\left(1+\frac{\rho_{p}|h|^{2}}{1+\rho_{J}\left|\boldsymbol{g}_{A}^{\mathrm{H}} \hat{\boldsymbol{g}}_{A}\right|^{2}}\right)\right] \\
& +\left(1-\frac{T_{\bar{B}}}{T_{B}}\right) \mathbb{E}\left[\log _{2}\left(1+\rho_{p}|h|^{2}\right)\right] .
\end{aligned}
$$

The term inside the first expectation is an upper bound on the capacity of the primary link, when jamming is present. $\frac{T_{\bar{B}}}{T_{B}}$ is the fraction of time the jammer beamforms to terminal $A$, when terminal B is transmitting, i.e., when the jamming does damage, as discussed in Section III-A. The rest of the time is wasted by beamforming to A while A is transmitting. This means that for a fraction $\left(1-\frac{T_{\bar{B}}}{T_{B}}\right)$ of the time, B transmits undisturbed, hence the normal SISO capacity expression in the second expectation. 


\section{Simulations}

There are a number of different parameters that affect the rate achieved by the primary link. All simulations use the path loss exponent $\alpha=3.8$ and the normalized transmit power of the primary link, $\rho_{p}=7 \mathrm{~dB}$ is fixed. It is important to note that the value of $\rho_{p}$ does not only affect the capacity of the primary link. The higher the transmit power of the primary link, the higher SNR at the jammer, which leads to a more efficient beamforming, and therefore a higher degradation of the primary link when jamming is applied. That is, even though the capacity of the primary link without jamming increases with $\rho_{p}$, the capacity of the jammed link can actually decrease.

The relative distance, $d_{r}$, is the ratio between the distance between the terminals in the primary link and the distance from the jammer to terminal A. This parameter, similarly to $\rho_{p}$, affects the SNR at the jammer. The further away the jammer is, the worse performance of the jammer.

The number of coherence blocks, $K$, that the jammer listens to is also a parameter that affects the ability to accurately estimate the frame timing. The longer the jammer listens, the better the estimate, but to be somewhat realistic this number cannot be too high, since the terminals are able to communicate freely, without being jammed, as long as the jammer is listening. In addition, the clock drift might also become a problem, if the jammer listens for too long.

The first simulation (see Figure 3) shows the performance of the proposed jammer, compared to an omnidirectional jammer and a genie jammer. The genie jammer knows the true frame timing as well as the true channel to terminal A. These two benchmark jammers are two extremes, one applies perfect beamforming, the other does not beamform at all. The simulation is run with the jammer having $M=100$ antennas, at relative distance $d_{r}=3$, and listening to $K=50$ coherence blocks. The simulations are shown for jammer power advantage $\rho_{J} / \rho_{p}$ from $-20 \mathrm{~dB}$ to $20 \mathrm{~dB}$ in Figure 3.

The performance of the proposed jammer is very close to the genie jammer, less than one $\mathrm{dB}$ difference. Comparing the proposed jammer with the omnidirectional jammer, it can be seen that the proposed jammer can do the same damage to the primary link as the omnidirectional jammer with close to 18 $\mathrm{dB}$ less output power. Another thing to note is that when the jammer and the primary link have the same output power, the damage that the omnidirectional jammer causes is minimal, the capacity of the primary link only drops about $1 \%$. Compare this to the proposed jammer, where the maximum rate of the primary link drops to less than $30 \%$ of its nominal capacity.

Figure 4 shows how the primary rate is affected when the jammer is placed at different relative distances from terminal A. Here, the jammer has no power advantage over the primary link, i.e $\rho_{J}=\rho_{p}$.

A few observations can be made from looking at the Figure 4. First, when the jammer is close to the primary users, the jammer performance is close to perfect. Second, for large relative distances, the proposed jammer performs no better

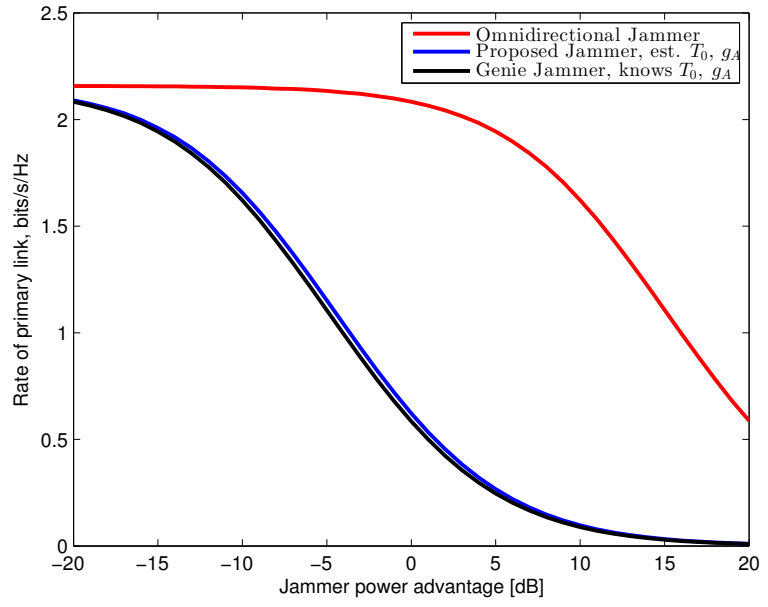

Fig. 3. Rate of the primary link as a function of jammer power advantage $\rho_{J} / \rho_{p}$. The jammer's parameters are: $M=100$ antennas, $K=50$ blocks, and relative distance $d_{r}=3$.

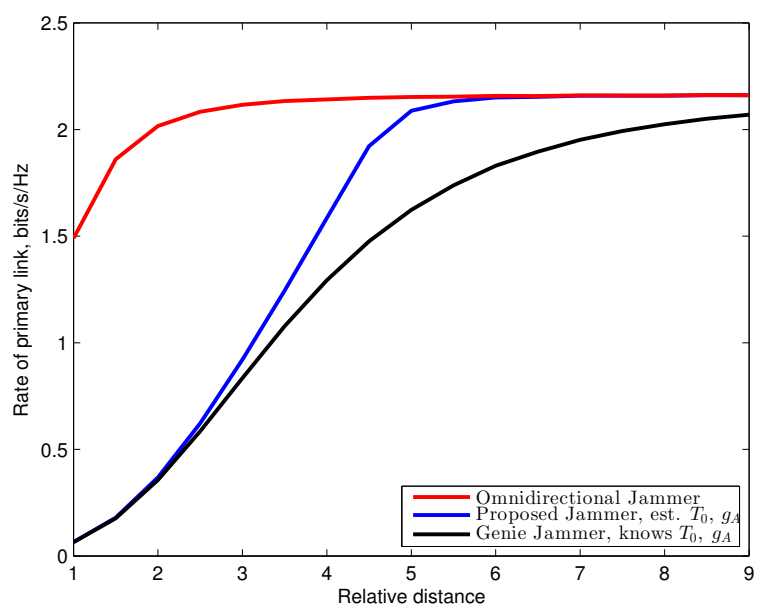

Fig. 4. Rate of the primary link as a function of relative distance $d_{r}$. The jammer's parameters are: $M=100$ antennas, $K=50$ blocks, and jammer power advantage $\rho_{J} / \rho_{p}=0 \mathrm{~dB}$.

than the omnidirectional jammer. The distance between the jammer and terminal A obviously degrades the performance of the jammer due to the increased attenuation of the jammed signal, but this alone does not explain the rapid deterioration of the proposed jammer. The effect of increased attenuation of the jamming signal on its own can be seen on the performance of the genie jammer. The distance between terminal A and the jammer affects the received signal power at the jammer. The further away the jammer is, the lower the SNR at the jammer, and at some point, the received signal will mostly consist of noise. This means that the frame timing will be difficult to estimate, and as a consequence, the channel estimates will be poor. If the jammer is very far away, the dominating eigenvector of the sample covariance matrix will be a random vector, associated with the noise $\boldsymbol{w}$.

How the number of antennas at the jammer affects the 
performance can be seen in Figure 5. The beamforming ability improves for each antenna that is added to the jammer. Ideally, the beamformed noise will scale linearly with the number of antennas, $M$, as the transmitted signal from the jammer experiences an array gain equal to $M$.

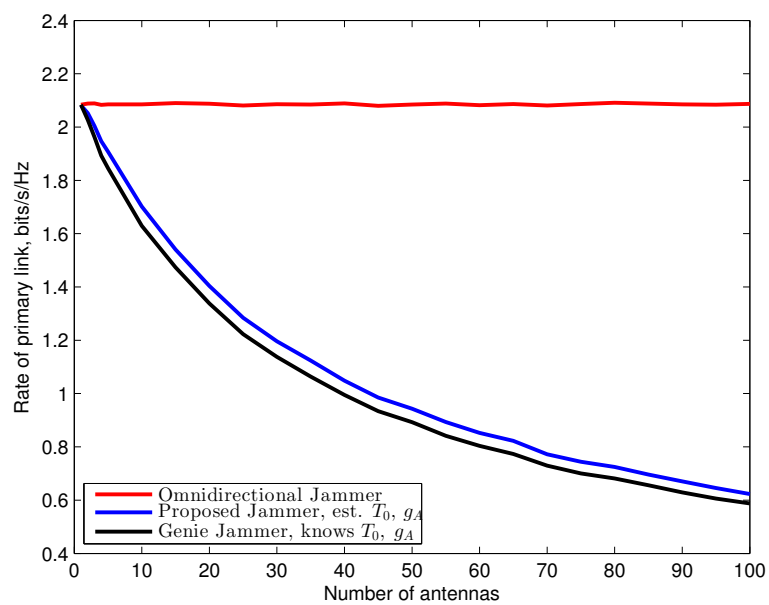

Fig. 5. Rate of the primary link as a function of number of antennas $M$. The jammer's parameters are: relative distance $d_{r}=3, K=50$ blocks, and jammer power advantage $\rho_{J} / \rho_{p}=0 \mathrm{~dB}$.

As stated before, an incorrect estimate of $T_{0}$ can greatly degrade the jamming performance. There are two main factors that contributes to how well the frame timing can be estimated, the first being the SNR observed at the jammer, and the second how many coherence blocks the jammer listens. Figure 6 shows how the relative distance affects the accuracy of the $T_{0}$ estimate. Here, the jammer has 100 antennas and the jammer listens to 50 coherence blocks. It can be seen that as long as the SNR at the jammer is good enough, the jammer estimates the frame timing quite well for this scenario. However, just as one can suspect from looking at Figure 4 there is a threshold when the SNR is just too low to estimate the frame timing accurately. The distribution of the errors can be seen going from a very peaky normal distribution, to a uniform distribution from $-\tau_{C} / 4$ to $\tau_{C} / 4$. Similar results can be seen when comparing the accuracy of the frame timing when listening to different number of coherence blocks. The distribution is close to normal when few blocks are used, but gets more peaky as the number of blocks increases. To be able to get this performance, the jammer needs to be close enough to the terminal to not have low SNR.

\section{CONCLUSION}

For the proposed jammer to be able to disrupt communication more efficiently than an omnidirectional jammer, estimation of the frame timing is crucial. A poorly estimated frame timing deteriorates the jamming performance substantially. There is a threshold, most notably in the relative distance after which the frame timing estimate proposed is not accurate. If certain conditions are met, the jammer can successfully

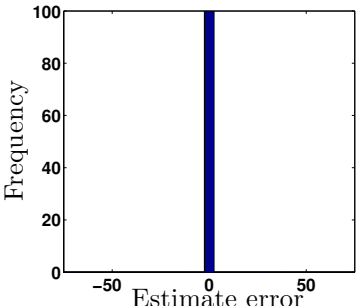

(a) $d_{r}=1$

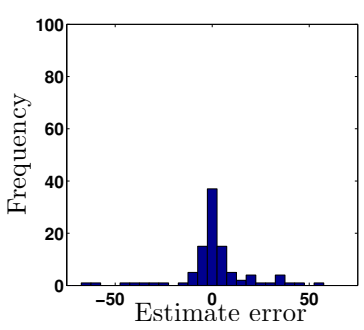

(c) $d_{r}=5$

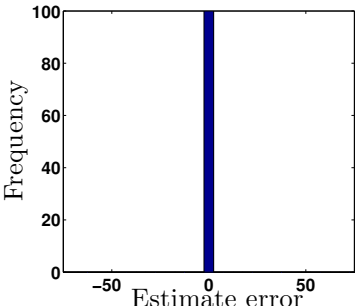

(b) $d_{r}=3$

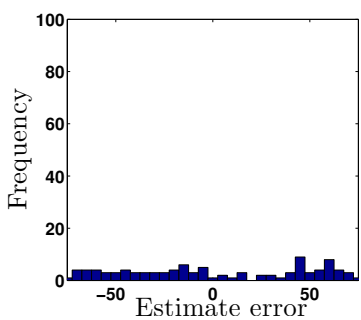

(d) $d_{r}=10$
Fig. 6. Estimation errors $\left(T_{0}-\hat{T}_{0}\right)$ for the frame timing for different relative distances. The vertical axis shows the frequency of the estimation errors (divided in bins of size 5 , symmetric around 0 ).

estimate the channel blindly, without any assumption of the structure of the channel. Future work will include extending the jammer to a multi-user setup and also how to handle wideband systems and frequency hopping.

\section{REFERENCES}

[1] T. Marzetta, "Noncooperative cellular wireless with unlimited numbers of base station antennas," IEEE Transactions on Wireless Communications, vol. 9, no. 11, pp. 3590-3600, November 2010.

[2] Mammoet project. [Online]. Available: http://mammoet-project.eu/

[3] Green touch. [Online]. Available: http://www.greentouch.org/

[4] E. Larsson, O. Edfors, F. Tufvesson, and T. Marzetta, "Massive MIMO for next generation wireless systems," IEEE Communications Magazine, vol. 52, no. 2, pp. 186-195, February 2014.

[5] F. Rusek, D. Persson, B. K. Lau, E. Larsson, T. Marzetta, O. Edfors, and F. Tufvesson, "Scaling up mimo: Opportunities and challenges with very large arrays," IEEE Signal Processing Magazine, vol. 30, no. 1, pp. 40-60, Jan 2013.

[6] H. Yang and T. Marzetta, "Total energy efficiency of cellular large scale antenna system multiple access mobile networks," in Online Conference on Green Communications (GreenCom), 2013 IEEE, Oct 2013, pp. 2732.

[7] E. Björnson, J. Hoydis, M. Kountouris, and M. Debbah, "Hardware impairments in large-scale MISO systems: Energy efficiency, estimation, and capacity limits," CoRR, vol. abs/1305.4651, 2013. [Online]. Available: http://arxiv.org/abs/1305.4651

[8] C. Shepard, H. Yu, N. Anand, E. Li, T. Marzetta, R. Yang, and L. Zhong, "Argos: Practical many-antenna base stations," in Proceedings of the 18th Annual International Conference on Mobile Computing and Networking, ser. Mobicom '12. New York, NY, USA: ACM, 2012, pp. 53-64. [Online]. Available: http://doi.acm.org/10.1145/2348543.2348553

[9] J. A. Volpe, Vulnerability Assessment of the Transportation Infrastructure Relying on the Global Positioning System. Department of Transportation, 2001. [Online]. Available: http://books.google.se/books?id=NZu-SgAACAAJ

[10] Göteborg 2001 : betänkande / av Göteborgskommitten., ser. Statens offentliga utredningar: 2002:122. Stockholm : Fritzes offentliga publikationer, 2002 (Stockholm : Elander Gotab), 2002.

[11] National telecommunications and information administration. [Online]. Available: http://www.ntia.doc.gov/files/ntia/va_tech_response.pdf 